\title{
Concept Forest: A New Ontology-assisted Text Document Similarity Measurement Method
}

\author{
James Z. Wang William Taylor \\ School of Computing \\ Clemson University, Box 340974 \\ Clemson, SC 29634-0974, USA \\ +1-864-656-7678 \\ \{jzwang,wptaylo\}@cs.clemson.edu
}

\begin{abstract}
Although using ontologies to assist information retrieval and text document processing has recently attracted more and more attention, existing ontologybased approaches have not shown advantages over the traditional keywords-based Latent Semantic Indexing (LSI) method. This paper proposes an algorithm to extract a concept forest $(C F)$ from a document with the assistance of a natural language ontology, the WordNet lexical database. Using concept forests to represent the semantics of text documents, the semantic similarities of these documents are then measured as the commonalities of their concept forests. Performance studies of text document clustering based on different document similarity measurement methods show that the CF-based similarity measurement is an effective alternative to the existing keywords-based methods. In particular, this CFbased approach has obvious advantages over the existing keywords-based methods, including LSI, in processing text abstracts or in $P 2 P$ environments where it is impractical to collect the entire document corpus for analysis.
\end{abstract}

\section{Introduction}

Currently, keywords-based techniques are commonly used in various information retrieval and text mining applications. Among them, Vector Space Model (VSM) [1] and Latent Semantic Indexing (LSI) [2] are the most widely adopted. Using VSM, a text document is represented by a vector of the frequencies of terms appearing in this document. The similarity between two text documents is measured as the cosine coefficient between their term frequency vectors. However, a major drawback of the keywords-based VSM approach is its inability of handling the polysemy and synonymy phenomena of the natural language. As meanings of words and understanding of concepts differ in different communities, different users might use the same word for different concepts (polysemy) or use different words for the same concept (synonymy). Thus, matching only keywords may not accurately reveal the semantic similarity among text documents or between search criteria and text documents due to the heterogeneity and independency of data sources and data repositories. For example, the keyword "java" can represent three different concepts: coffee, an island, or a programming language, while keywords "dog" and "canine" may represent the same concept in different documents.

LSI tries to overcome the limitation of VSM by using statistically derived conceptual indices to represent text documents and queries. LSI assumes that there is an underlying latent structure in word usage that is partially obscured by variability of word choice and tries to address the polysemy and synonymy problems through modeling the co-occurrence of keywords in documents. Though earlier studies contend that LSI may implicitly reveal concepts through the co-occurrence of keywords, we found that the co-occurrence of keywords may not necessarily mean their contextuality in the document, especially in multi-disciplinary research papers. This is exactly why using LSI-based tools to extract terms from commercial web documents, which may contain ads, headlines, and news feeds, is a questionable practice. On the other hand, how to map the LSI-based conceptual index into the underlying concept is not clear, making it difficult to visualize the text mining results. In addition, some text document archives, such as MEDLINE database [3] and web blogging entries, contain primarily short articles or abstracts instead of long papers. These short documents may not provide sufficient cooccurrence information for LSI-based semantic similarity measurement. Furthermore, in dynamic environments, such as live news feeds or P2P systems, it is impractical to collect the entire document corpus for analysis.

In this paper, to address the weaknesses of existing keywords-based approaches, we propose an ontology- 
assisted text document similarity measurement method by building a concept forest to represent the semantics of a text document. The rest of this paper is organized as follows. We first discuss the existing ontology-based approaches and their weaknesses in section 2 and then discuss our ontology-assisted concept forest construction algorithm and the associated similarity measurement method in section 3 . In section 4 , we cluster various text document corpuses based on similarity values obtained by different methods to validate the advantages of our CF-based approach. Finally we give our conclusion and discuss the future work in section 5 .

\section{Background and existing approaches}

Recently, to address the problems in keywords-based approaches, many studies tried to use ontologies to assist information retrieval and text document processing. These ontology-based approaches can be divided into two categories. One category of ontology-based methods [4, 5, 6, 7] apply machine learning methods, such as clustering analysis and fuzzy logic, to construct ontologies from text documents and, then, use these ontologies to assist information retrieval and text document processing [8,9]. However, these methods require analyzing the entire document corpus to construct a good ontology, and the performance of information retrieval and text document processing depends on how good the constructed ontologies are. During the corpus analysis, terms rarely appearing in the document corpus are often ignored because of their low frequencies of occurrence. However, high information content of these rare terms is valuable for information retrieval according to information theory. Ignoring these terms in the constructed ontologies may affect the performance of information retrieval and text document processing. Nonetheless, these ontology-based methods have not been fully evaluated against the keywords-based LSI method, arguably the best keywords-based method.

Another group of ontology-based methods utilize an existing ontology, such as WordNet [10], to assist information retrieval and text document processing. These methods use three different approaches to take advantage of the existing ontological knowledge. The first approach $[11,12]$ involves using WordNet to find synonyms or hypernyms of terms to improve the performance of information retrieval and text document processing. However, this approach may introduce "noise" by adding semantic content that is not present in the document corpus. For instance, given a document about "beef" and a document about "pork", a hypernymbased method may use "meat" to replace "beef" and "pork" because two terms have a common hypernym "meat". This approach over-simplifies or overgeneralizes the problem, making it impossible to distinguish documents containing "beef" from documents containing "pork". Another problem with this approach is that it does not perform word sense disambiguation. Instead, all synonyms or hypernyms related to a keyword are used to replace the keyword. These weaknesses often lead to disappointing information retrieval and text document processing performance [13, 14].

The second approach focuses on word sense disambiguation $[15,16,17,18]$ to address the synonymy and polysemy problems in natural language processing. However, this approach tries to determine an exact sense for a term, often resulting in misclassification of terms. This approach also ignores the impact of the semantic similarities and relationships among different terms in the same text document on the performance of information retrieval and text document processing.

To address the problems in the first two approaches, the third approach applies various techniques [19, 20, 21, $22]$ to discover the semantic similarities and relationships of terms and use them to enhance the keywords-based information retrieval and text document processing methods, such as VSM. However, the techniques used to discover the term relationships and similarities have their weaknesses. Sedding [19] used a naive, syntax-based disambiguation approach by assigning each word a partof-speech (POS) tag and by enriching the "bag-of-words" data representation, which extracts synonyms and hypernyms from WordNet to use in document clustering. Unfortunately, this study found that including synonyms and hypernyms, disambiguated only by PoS tags, does not improve the effectiveness of text document clustering. The authors attributed this underperformance to the noise introduced by incorrect senses retrieved from WordNet and concluded that disambiguation by PoS alone is insufficient to reveal the full potential of including background knowledge in information retrieval and text document processing. To further investigate this issue, Simone [20] proposed a document search technique that uses other methods, in addition to POS tagging, to cluster search results into meaningful categories according to the words that modify the original search term in the text document. This work focuses on determining if the antonymy relation, instead of synonyms and hypernyms, could be used on the modifiers found in documents to decompose a set of search results into a hierarchy of sub-clusters. Unfortunately, their experimental studies again suggest that this approach cannot improve the performance of information retrieval.

While these two studies [19, 20] suggest exploiting term relationships or similarities using WordNet may not improve the performance of information retrieval and text document processing, other studies using different methods imply that it is possible to use term relationships 
or similarities to improve the performance of the keywords-based VSM. Huang [21] used a guided selforganization map (SOM), a result of merging statistical methods, competitive neural models, and semantic relationships obtained from WordNet, to improve the performance of the traditional VSM. However, certain human involvement is required to build the guided SOM. Jing [22] calculates a mutual information matrix for all terms in the documents based on information obtained from WordNet and uses the mutual information to enhance the keywords-based VSM method. However automatically computing term mutual information (TMI) is sometimes problematic and may lead to wrong conclusions about the quality of the learned mutual similarity [23]. Even though using SOM and TMI can improve the performance of the keywords-based VSM, their performance in comparison with LSI, the best keywords-based method, has not been investigated. Furthermore, these methods require analyzing the entire document corpus as VSM and LSI do.

To address the problems in existing ontology-based methods, we propose a new ontology-assisted method to measure the semantic similarity of text documents. This new method constructs a concept forest (CF) from a text document, based on the co-occurrence of terms and their semantic relationships found in WordNet. Using the CF to represent the semantics text documents, we propose a simple method to measure the semantic similarity of two text documents. A unique feature of our proposed CFbased method is that we derive the concept forest based only on analyzing the co-occurrences and relationships of terms within a single document. Conversely, existing approaches all require analyzing the entire text document corpus to determine the semantic similarity of two text documents. Therefore, our CF-based method is a practical alternative to the existing information retrieval and text document processing methods in dynamic environments such as P2P systems and live news feeds, where it is impractical to collect the entire document corpus for analysis.

\section{Concept Forest and Semantic Similarity}

Our CF-based method includes three steps: concept forest construction, semantic content purification, and similarity measurement.

\subsection{Concept Forest Construction}

We use WordNet [10] to assist our concept forest construction. WordNet is a large lexical database of English words, in which nouns, verbs, adjectives and adverbs are grouped into sets of cognitive synonyms (synsets) with each synset representing a distinct concept. Synsets are interlinked by means of conceptual and lexical relations. There are approximately 150,000 words organized in over 115,000 synsets in WordNet. Every synset contains a group of synonymous words or collocations with different senses (concepts) of a word being in different synsets. Most synsets are connected to other synsets through semantic relations, such as hypernym, hyponym, etc. The dominant semantic relationship in WordNet is hypernym, the "is-a" relationship. Most nouns and verbs are organized into hierarchies, defined by hypernym or "is-a" relationships. For example, Figure 1 depicts the hypernym hierarchy for the first sense of the word "dog".

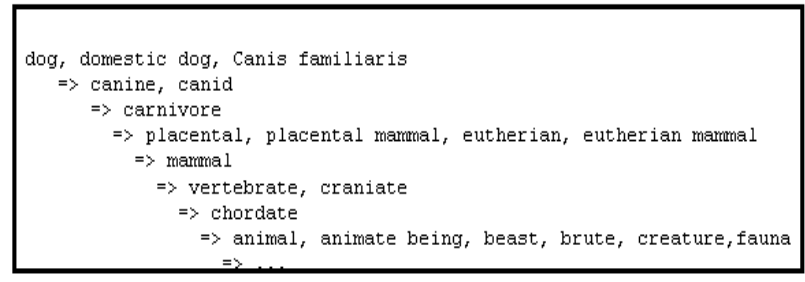

Figure 1: Hierarchy of Hypernym Relationships.

Given a text document, we first extract all keywords and their occurrence frequencies from the document, excluding stop words such as pronouns, common verbs, common nouns, adjectives, and frilly words. These words add little or no value in determining the document's semantic content according to previous studies [1, 2]. We then use a simple WordNet morphology interface (function morphstr()) to stem these keywords, i.e., to map inflected (or sometimes derived) words to their stem, base or root form. For instance, "cared", "cares", and "caring" are all mapped to the root word "care". After word stemming, we determine the proper synset for a word based on the co-occurrence of terms in the document and the semantic relationships of senses defined in WordNet.

In WordNet, each set of synonyms (synset) shares some common properties, such as a gloss (or dictionary) definition, indexed by a unique ID (called synsetID). However, one word may be related to several synsets due to the polysemy of the natural language. For instance, the word "java" has three different senses: (1) Coffee, cafe (synsetID: 67893543); (2) Programming, Programming Language (synsetID: 71154118); (3) An Island (synsetID: 82735541). Therefore, simply retrieving all senses of the stemmed words to represent the semantic content of a document introduces a lot of "noise" [13, 14]. To address this issue, for any stemmed word obtained from a document, we only use the sense that clearly represents the concept of the word in this document for our concept forest construction.

Our procedure checks every pair of stemmed words obtained from the text document to determine whether 
there are semantic relationships between their senses defined in WordNet. We only consider the hypernym relationship in this study because considering only the hypernym relationship is adequate for measuring the semantic similarity of documents due to the dominance of the hypernym relationship among the terms in text documents according to our experimental studies.

Given two terms (T1 and T2) obtained from the same text document, if their respective synsets $\mathrm{S} 1$ and $\mathrm{S} 2$ have a hypernym relationship, the synsetIDs of S1 and S2 are used to represent the concepts of $\mathrm{T} 1$ and $\mathrm{T} 2$ respectively, and other senses of $\mathrm{T} 1$ and $\mathrm{T} 2$ will be discarded. Meanwhile, a "is-a" relationship link is formed between the synsetIDs of S1 and S2. This process completes when all pairs of stemmed words are investigated. For instance, given a document containing words "disease", "sickness", "influenza", "drug" and "medicine", we can construct a concept tree for terms "disease", "sickness" and "influenza" using "is-a" relationship link based on the hypernym relationship among these terms as shown in Figure 2. Similarly, a concept tree can be built for terms "drug" and "medicine". These two concept trees form a concept forest depicted in Figure 3. We note that the terms instead of their related synsetIDs are shown in the concept forest for demonstration only. In actual concept forests, the synsetIDs are used to represent the concepts whenever possible. We also note that a concept tree may contain only a single stemmed word.

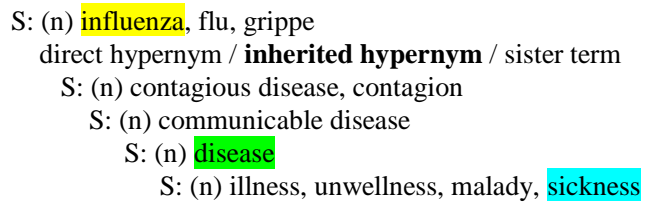

Figure 2: Hypernym hierarchy for terms "influenza", "disease" and "sickness".
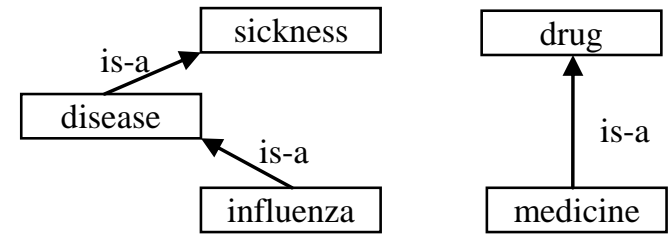

Figure 3: Concept forest derived from terms "influenza", "disease", "sickness", "drug" and "medicine".

Unlike some existing approaches [1,2], which use all terms in all synsets of the stemmed words to represent the semantic content of a document, we treat keywords differently according to their synset properties and the semantic relationships among the synsets of keywords. If a keyword has only one sense, its synsetID will be used in the concept forest. If a keyword has more than one sense and no other keyword's senses have semantic relationships with this keyword's senses, then this keyword will be kept as its original stemmed word in the concept forest since we cannot disambiguate the word sense. Finally, if a keyword has many senses, and one or more senses have semantic relationships with the senses of other keywords in the text document, only the synsetIDs of the senses having semantic relationships with the senses of other keywords will be kept in the concept forest. Other senses of this keyword will be discarded since they are irrelevant to the semantic content of the text document.

\subsection{Semantic Content Purification}

A concept forest constructed by method described in Section 3.1 may contain terms or synsetIDs that are not closely related to the main topics of the text document, and these terms or synsetIDs may sometimes introduce noise to information retrieval and text document processing. To address this issue, we use the frequencies of terms occurring in the text document to calculate a semantic content rate (SCR) for a concept tree in the concept forest.

Each stemmed word obtained from a text document has an associated word frequency value corresponding to the number of occurrences that this word was found in the text document. When a stemmed word is mapped to a particular synsetID during the CF construction, the associated word frequency value is transferred to the synsetID. If several stemmed words are mapped to the same synsetID, the word frequency value of this synsetID is the sum of the word frequency values of these associated words. We further define the semantic content weight for a concept tree as the sum of the word frequency values of all its associated synsetIDs. For a single-node tree, its semantic content weight is the word frequency value of this single node.

Assuming the semantic content weights of concept trees in a concept forest are $w_{1}, w_{2}, \ldots, w_{n}$, respectively, the semantic content rate of concept tree $i$ is defined as:

$$
S C R_{i}=w_{i} / \sum_{j=1}^{n} w_{j}
$$

The SCR values in a concept forest indicate the semantic organization of the associated text document. A concept forest obtained from a clearly and concisely written single-topic abstract may contain a concept tree having an SCR value greater than $75 \%$, while the concept forest obtained from a long multiple-topic text document may contain several concept trees with much smaller SCR values. To purify the semantic content of a concept forest, we use a threshold (e.g., 5\%) to filter out concept trees with low SCR values. Any concept tree whose SCR value falls below this threshold will be removed from the final purified concept forest. 


\subsection{Semantic Similarity Measurement}

Using a concept forest to represent the semantic content of a text document, the semantic similarity of two text documents can be determined by comparing their concept forests. Formally, an concept forest is defined as a Directed Acyclic Graph (DAG): $\mathrm{CF}=[T, E, R]$, where $T=\left\{t_{1}, t_{2}, \ldots, t_{n}\right\}$ is a set of stemmed words or synsetIDs, and $E=\left\{\mathrm{e}_{1}, e_{2}, \ldots, e_{m}\right\}$ is a set of edges connecting synsetIDs with relationships defined in $R=\left\{r_{1}, r_{2}, \ldots\right.$, $\left.r_{k}\right\}$. Specifically, an edge $e_{i}$ is defined as a triplet $\left[t_{i l}, t_{i 2}\right.$, $r_{j}$ ] where $t_{i 1}, t_{i 2} \in T$ and $r_{j} \in R$. In addition, two terms can be linked by only one relationship, that is,

$$
\forall l \neq k,\left[t_{i}, t_{j}, r_{k}\right] \in E \Rightarrow\left[t_{i}, t_{j}, r_{l}\right] \notin E .
$$

For instance, the forest concept in Figure 3 can be represented as $\mathrm{CF}=[\{$ "disease", "sickness", "influenza", "drug", "medicine"\}, \{["influenza", "disease", "is-a"], ["disease", "sickness", "is-a"], ["medicine", "drug", "isa"] $\},\{$ "is-a" $\}]$.

Given two documents $\mathrm{D}_{1}$ and $\mathrm{D}_{2}$, and their concept forests $\mathrm{CF}_{1}=\left[\begin{array}{lll}T_{1}, & E_{1}, & R_{1}\end{array}\right]$ and $\mathrm{CF}_{2}=\left[\begin{array}{lll}T_{2}, & E_{2}, & R_{2}\end{array}\right]$ respectively, determining the semantic similarity of these two documents needs to consider the similarities of the term sets, edge sets, and relationship sets in their concept forests. However, we use only the hypernym ("is-a") relationship to construct the $\mathrm{CF}$ and thus the relationship set $\mathrm{R}$ is the same for all CFs. On the other hand, the selection of terms during the $\mathrm{CF}$ construction implies their relationships. Therefore, we calculate the semantic similarity of two text documents by simply comparing the similarity of the term sets $\left(\mathrm{T}_{1}\right.$ and $\left.\mathrm{T}_{2}\right)$ in their concept forests, hoping this simple measurement is sufficient for information retrieval and text document processing. That is:

$$
\operatorname{Sim}\left(D_{1}, D_{2}\right)=\frac{\left|T_{1} \cap T_{2}\right|}{\left|T_{1} \cup T_{2}\right|}
$$

\section{Experimental Studies}

To evaluate whether our obtained concept forest can represent the semantic content of a document, we cluster text documents based on their semantic similarity values calculated by Equation 2. The clustering results are then compared with the results of document clustering based on VSM and LSI respectively.

\subsection{Text Document Corpus}

As in many previous studies, we derive our document corpuses from Reuters-21578 Text Categorization Collection in UCI KDD archive [24]. The Reuters-21578 dataset is a collection of documents that appeared on Reuters' newswire in 1987. The documents were assembled and indexed with categories. This dataset consists of approximately 21,500 files covering 132 (possibly overlapping) categories with the file size per article ranging from 12 to 900 words.

As we discussed previously, LSI is not efficient in information retrieval and text document processing for short text documents due to the insufficient cooccurrence information within the short documents. We want to study whether our CF-based text document similarity measurement method can address this issue so that it can be used for information retrieval in text abstract databases, such as MEDLINE database. Therefore, we intentionally select text documents containing less than 400 words. As shown in Table 1, four text document corpuses containing 50 to 500 documents are selected for our experimental studies.

Table 1: Selected text document corpuses

\begin{tabular}{|c|l|}
\hline Corpus & Corpus Characteristics \\
\hline C-1 & $\begin{array}{l}\text { 50 Documents, 2 categories (Oil, Nat-Gas), } \\
\text { 25 documents in each category. }\end{array}$ \\
\hline C-2 & $\begin{array}{l}\text { 100 Documents, 2 categories (Coffee, Sugar), } \\
50 \text { documents in each category. }\end{array}$ \\
\hline C-3 & $\begin{array}{l}\text { 200 Documents, 4 categories (Grain, Wheat, } \\
\text { Ship, Crude), 50 documents in each category. }\end{array}$ \\
\hline C-4 & $\begin{array}{l}\text { 500 Documents, 2 categories (Wheat, Grain), } \\
\text { 250 documents in each category. }\end{array}$ \\
\hline
\end{tabular}

\subsection{Performance Evaluation Method}

Although many studies used K-means clustering algorithm or its variants for text document clustering [13, 14], $\mathrm{K}$-means algorithm is not suitable for text document clustering using our CF-based similarity measurement because it does not make sense to calculate a mean similarity among a set of documents. Therefore, an agglomerative hierarchical clustering algorithm is used in our performance study.

Given a text document corpus, each document initially belongs to its own individual cluster. We set the initial similarity threshold to be 1 and decrease the threshold with a small interval so that documents with similar semantics will be gradually merged into the same group. Since we already know the categories from which each document was obtained, the document clustering process stops when the majority of documents from different categories fall into their respective clusters and further decreasing the threshold will cause clusters containing documents primarily from two different categories merged into one cluster. After the document clustering, we calculate the clustering accuracy as the number of documents correctly clustered into their categories divided by the total number of documents. 
Besides clustering text documents based on our CFbased similarity measurement method, we also perform the document clustering using VSM and LSI as the document similarity measurement methods. For VSM, the cosine coefficients of the document vectors are used as the similarity measures. For LSI, we calculate the rank $k$ approximation of term vector for each document and calculate their similarities using cosine coefficients of their term vectors. Then we use these similarity values to cluster the text documents. We repeat the same process under different $k$ values and report the best clustering results for LSI.

\subsection{Performance Results}

We conducted our experimental studies on a DELL desktop computer equipped with a $1.0 \mathrm{GHz}$ Intel Pentium IV processor and $512 \mathrm{MB}$ RAM, running the Red Hat Enterprise Linux. We cluster the text document corpuses listed in Table 1 based on three different similarity measurement methods, VSM, LSI, and CF-based method. The accuracies of text document clustering using different methods are listed in Table 2 . In addition to the clustering accuracy, we also observe the total time needed to complete the corpus analysis and document clustering. The results are reported in Figure 4.

Table 2: Clustering accuracies on text corpuses listed in Table 1

\begin{tabular}{|c|c|c|c|}
\hline & VSM & LSI & CF \\
\hline C-1 & $64 \%$ & $64 \%$ & $74 \%$ \\
\hline C-2 & $50 \%$ & $62 \%$ & $80 \%$ \\
\hline C-3 & $25 \%$ & $34 \%$ & $48 \%$ \\
\hline C-4 & $50 \%$ & $56.8 \%$ & $68 \%$ \\
\hline
\end{tabular}

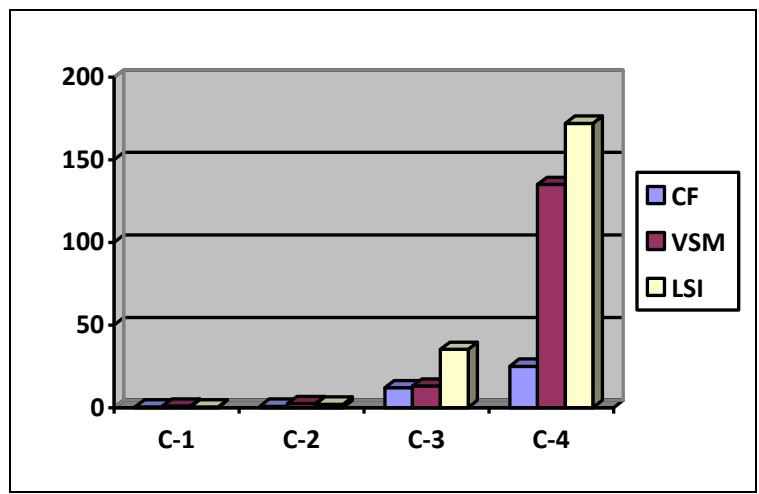

Figure 4: Total time (minutes) needed to complete the corpus analysis and text document clustering using different methods on text corpuses listed in Table 1.

The performance results in Table 2 show that the accuracy of document clustering based on our CF-based similarity measurement is much better than that based on
VSM or LSI. On the other hand, the clustering accuracies based on LSI are better than those based on VSM. The execution time depicted in Figure 4 exhibit the runtime efficiency of our CF-based text document processing method. The total time spent on corpus analysis and document clustering using the CF-based method is much less than that based on VSM or LSI.

\section{Conclusion and Future Studies}

In this paper, we propose a novel algorithm to extract a concept forest (CF) from a text document with the assistance of a natural language ontology, WordNet lexical database. Using concept forests to represent the semantics of text documents, we measure the semantic similarity of two text documents by simply comparing the term sets in their respective concept forests. This CFbased similarity measurement does not require analyzing the entire document corpus, an advantage over most existing document similarity measurement methods, including the popular VSM and LSI. This unique advantage allows our CF-based text document similarity measurement method to be used P2P environments where collecting the entire document corpus for analysis is impractical.

Our experimental studies also show that the CF-based text document similarity measurement method performs much better than both VSM and LSI methods when document sizes are relatively small. Furthermore, our CF-based document similarity measurement method is much more efficient regarding the total execution time used for corpus analysis and document clustering. Therefore, we believe the CF-based approach is a practical alternative to the existing keywords-based methods for information retrieval and text mining in text abstract databases, such as MEDLINE.

We are currently designing a graph-matching-based method to compare the similarity of two concept forests, hoping to provide a more sophisticated text document similarity measurement and improve the text document clustering accuracy. We are also implementing a CFbased information retrieval system to effectively retrieve text abstracts from MEDLINE database.

\section{References}

[1] G. Salton, A. Wong, and C. S. Yang (1975), A Vector Space Model for Automatic Indexing, Communications of the ACM, vol. 18, no. 11, pages 613-620.

[2] Deerwester, S., Dumais, S. T., Furnas, G. W., Landauer, T. K., \& Harshman, R. (1990). Indexing by latent semantic analysis. Journal of the American Society for Information Science, 41(6), 391-407. 
[3] MEDLINE

Fact

Sheet, http://www.nlm.nih.gov/pubs/factsheets/medline.htm l

[4] Lee, C.S., Jian, Z.W. and Huang, L.K., A fuzzy ontology and its application to news summarization. IEEE Transactions on Systems, Man, and Cybernetics, Part B: Cybernetics. Volume 35, Issue 5. pp. 859-880.

[5] Lipika Dey, Ashish Chandra Rastogi, Sachin Kumar, Generating Concept Ontologies through Text Mining, 2006 IEEE/WIC/ACM International Conference on Web Intelligence (WI'06), pp. 23-32, 2006.

[6] O. S. Chin, N. Kulathuramaiyer, A. W. Yeo, Automatic Discovery of Concepts from Text, IEEE/WIC/ACM International Conference on Web Intelligence (WI 2006), pp. 1046-1049, December 2006

[7] Blaz Fortuna, Dunja Mladenic and Marko Grobelnik, Semi-Automatic Construction of Topic Ontology, Conference on Data Mining and Data Warehouses (SiKDD 2005) at multiconference IS 2005.

[8] Navigli, R., Velardi, P. and Gangemi, A., Ontology learning and its application to automated terminology translation. IEEE Intelligent Systems. Volume 18, Issue 1. pp. 22-31.

[9] Sugumaran, V. and Storey, V.C., Ontologies for conceptual modeling: their creation, use, and management. International Journal of Data and Knowledge Engineering. Volume 42, Issue 3. pp. 251-271.

[10] Christine Fellbaum (ed.), WordNet: An Electronic Lexical Database. The MIT Press, May 1998.

[11]S. Scott and S. Matwin. Text Classification using WordNet Hypernyms. In S. Harabagiu, editor, Use of WordNet in Natural Language Processing Systems: Proceedings of the Conference, pages 38-44. Association for Computational Linguistics, Somerset, New Jersey, 1998.

[12]D. Koller, and M. Sahami, Hierarchically classifying documents using very few words, Proceedings of the 14th international Conference on Machine Learning ECML98, 1998.

[13]A. Kehagias, V. Petridis, V.G. Kaburlasos, and P. Fragkou, "A comparison of word- and sense-based text categorization using several classification algorithms," Journal of Intelligent Information Systems, 21(3), 2001.

[14]A. Hotho, S. Staab, and G. Stumme. Ontologies improve text document clustering. In Proceedings of the IEEE International Conference on Data Mining, pages 541-544, 2003.
[15]Dimitrios Mavroeidis et al., Word Sense Disambiguation for Exploiting Hierarchical Thesauri in Text Classification, A. Jorge et al. (Eds.): PKDD 2005, LNAI 3721, pp. 181-192, 2005.

[16] Youjin Chung and Jong-Hyeok Lee, Practical WordSense Disambiguation Using Co-occurring Concept Codes, Machine Translation (2005) 19: 59-82.

[17]Ernesto William De Luca, Andreas Nürnberger: Using clustering methods to improve ontology-based query term disambiguation. Int. J. Intell. Syst. 21(7): 693-709 (2006)

[18] Ying Liu, Peter Scheuermann, Xingsen Li, and Xingquan Zhu, Using WordNet to Disambiguate Word Senses for Text Classification, Y. Shi et al. (Eds.): ICCS 2007, Part III, LNCS 4489, pp. 780788, 2007.

[19] J. Sedding and D. Kazakov. WordNet-based Text Document Clustering. In Proc. of the Third Workshop on Robust Methods in Analysis of Natural Language Data (ROMAND), pp.104-113, Geneva, 2004.

[20] Thomas de Simone and Dimitar Kazakov. Using WordNet Similarity and Antonymy Relations to Aid Document Retrieval. Recent Advances in Natural Language Processing (RANLP 2005), 21-23 September 2005, Borovets, Bulgaria.

[21]Chihli Hung, Stefan Wermter and Peter Smith, Hybrid Neural Document Clustering Using Guided Self-Organization and WordNet, IEEE Intelligent Systems, Vol. 19, No. 2, pp. 68-77, 2004.

[22]L. Jing, L. Zhou, M. Ng and J. Huang, Ontologybased Distance Measure for Text Clustering, SIAM Text Mining 2006 Workshop.

[23] Marta Sabou, Learning Web Service Ontologies: an Automatic Extraction Method and its Evaluation, Ontology Learning and Population ( Editors: P.Buitelaar, P. Cimiano, B. Magnini), IOS Press, 2005

[24]Reuters-21578 Text Categorization Collection, http://kdd.ics.uci.edu/databases/reuters21578/reuter s21578.html 